\title{
Historia y producción científica de la investigación de la comunicación en España (1980-2009)
}

\author{
History and scientific production of communication research in Spain \\ (1980-2009)
}

Leonarda GARCÍA-JIMÉNEZ

Universidad de Murcia (España)

leonardagj@um.es

Recibido: 23 de septiembre del 2016

Aceptado: 14 de octubre 2016

\begin{abstract}
Resumen
En este texto desarrollaré cuáles han sido las principales tendencias en la producción científica española y a qué contextos académicos, sociales y epistemológicos responde dicha proliferación científica desde 1980 hasta 2009. Este trabajo fue presentado por primera vez en la VIII Bienal de Comunicación (México, 2011), donde expuse las principales tendencias de la investigación en España desde 1980 hasta 2006. Para esta publicación, las publicaciones en España han sido actualizadas hasta el año 2009, con la inclusión de 100 nuevas publicaciones correspondientes a los años 2007, 2008 y 2009. Para ello, se han manejado y explotado estadísticamente un total de 339 referencias (libros, artículos y capítulos de libro) publicadas entre 1980 y 2009. Dichas referencias han sido clasificadas en función de su objeto de estudio (metateoría, epistemología, elementos objetivos, subjetivos y práxicos y aportaciones teóricas generales, teniendo en cuenta, asimismo, cuáles han sido las etapas históricas en las que han sido publicadas. Esta explotación estadística mostrará la evolución de la investigación en España y cómo los diferentes contextos sociales/mediáticos, académicos y epistemológicos priorizaron unos objetos de estudio frente a otros.
\end{abstract}




\begin{abstract}
In this text I will develop which have been the main trends in Spanish scientific production and what academic, social and epistemological contexts this scientific proliferation responds to from 1980 to 2009. This work was presented for the first time at the VIII Biennial of Communication (Mexico, 2011), where I exposed the main research trends in Spain from 1980 to 2006. For this publication, the publications in Spain have been updated until 2009, with the inclusion of 100 new publications corresponding to the years 2007, 2008 and 2009. For Therefore, a total of 339 references (books, articles and book chapters) published between 1980 and 2009 have been statistically managed and exploited. Said references have been classified according to their object of study (metatheory, epistemology, objective, subjective and practical and general theoretical contributions, taking into account, also, what have been the historical stages as in which they have been published. This statistical exploitation will show the evolution of research in Spain and how the different social / media, academic and epistemological contexts prioritized some objects of study over others.
\end{abstract}

Palabras Clave: Producción científica; comunicación; investigación; España.

Keywords: Scientific production; communication; investigation; Spain.

\title{
1. Introducción
}

La investigación de la comunicación en España cuenta con más de tres décadas de producción científica, de desarrollo académico y de crecimiento exponencial de estudiantes y estudiosos enmarcados en este ámbito ${ }^{1}$. Tal y como apunta Donsbach (2006, p. 437) con respecto a la investigación en comunicación a nivel internacional, también podría decirse que la disciplina de la comunicación es la que más ha crecido en España en los últimos 30 años. Además, la contemporánea sociedad informacional, protagonizada por la convergencia mediática, los medios digitales, los contenidos multisoporte/multimedia y la proliferación de

\footnotetext{
${ }^{1}$ Agradezco sinceramente la colaboración de Noelia Belmonte García en la localización y recuperación de referencias bibliográficas. La actualización de este trabajo se hizo durante una estancia investigadora en la Universidad de Colorado subvencionada por la Fundación Séneca (Murcia, España). Su código de referencia es 18699/EE/12.
} 
terminales interactivas (tablets, ipads, smartphones, videoconsolas, etc.), nos está indicando que este crecimiento seguirá en aumento y que, por lo tanto, el campo de la comunicación sigue hoy más vivo que nunca, cuestión que ya preconizara a mediados de la década de los 50 Wilbur Schramm (1959) en su mítica disputa con Bernard Berelson (1959).

Tras más de tres décadas de investigación, podría decirse que el campo español ha alcanzado la madurez disciplinar. Sin duda, han sido claves el desarrollo institucional (facultades, revistas, grupos de investigación, etc.) (García Jiménez, 2007, p. 153) y el consiguiente tejido de redes estables de formación e intercambio. La proliferación institucional y científica ha generado otro síntoma que también refleja la madurez de la investigación española: el que cada vez más la comunidad epistémica se toma a sí misma como objeto de estudio. Al igual que es la persona adulta aquella que está en disposición de responder a preguntas existenciales sobre el sentido y la identidad, las disciplinas, solamente cuando son "adultas", pueden enfrentarse a este tipo de auto-reflexión metateórica.

Sobre la disciplina en España, de los precursores trabajos que realizara Jones (1997, 1998, 1999), Cáceres y Caffarel (1992), Moragas (1981 y 1988) o Rodrigo Alsina (2001), recientemente más investigadores están analizando las líneas de investigación y tendencias metodológicas y paradigmáticas de las ciencias de la comunicación en España (Martínez Nicolás, 2006 y 2009; Castillo y Carretón, 2010; Martínez Nicolás y Saperas, 2009; Rodrigo Alsina y García Jiménez, 2010 o García Jiménez, 2007). La formación española en nuestras facultades también está siendo objeto de exploración (Lozano y Vicente, 2010; García Avilés y García Jiménez, 2009).

En este texto desarrollaré cuáles han sido las principales tendencias en la producción científica española y a qué contextos académicos, sociales y epistemológicos responde dicha proliferación científica desde 1980 hasta 2009. Este trabajo fue presentado por primera vez en la VIII Bienal de Comunicación (México, 2011), donde expuse las principales tendencias de la investigación en España desde 1980 hasta 2006. Para esta publicación, las 
publicaciones en España han sido actualizadas hasta el año 2009, con la inclusión de 100 nuevas publicaciones correspondientes a los años 2007, 2008 y 2009. Para ello, se han manejado y explotado estadísticamente un total de 339 referencias (libros, artículos y capítulos de libro) publicadas entre 1980 y 2009. Dichas referencias han sido clasificadas en función de su objeto de estudio (metateoría, epistemología, elementos objetivos, subjetivos y práxicos y aportaciones teóricas generales (García Jiménez, 2007, p. 178 y ss.), teniendo en cuenta, asimismo, cuáles han sido las etapas históricas en las que han sido publicadas. Esta explotación estadística mostrará la evolución de la investigación en España y cómo los diferentes contextos sociales/mediáticos, académicos y epistemológicos priorizaron unos objetos de estudio frente a otros.

Por todo ello, en primer lugar, esbozaré brevemente las etapas de nuestra investigación, así como sus contextos sociales, académicos y epistemológicos ${ }^{2}$. Una vez planteada de manera muy sucinta la historia intelectual de la comunicología española, en segundo lugar, explotaré estadísticamente la investigación española en función de las etapas. Cuáles han sido los principales objetos de estudio en función de las dos etapas clave de la investigación española, es decir, las dos etapas que han visto eclosionar los estudios y análisis de la comunicación. Se trata de la etapa II (la del desarrollo, desde 1980 a 1995) y la etapa III contemporánea (la de la estandarización, a partir de 1996). Finalmente, basándome en la propia historia y trayectoria del caso español, planteo algunas medidas e iniciativas con el fin de potenciar el campo de la investigación iberoamericana.

En definitiva, este capítulo es una nueva aportación en la línea de exploración metateórica de la producción científica en España, con la esperanza de que el mejor conocimiento de lo que somos, sirva como plataforma con la que consolidar el espacio común de investigación iberoamericano.

\footnotetext{
${ }^{2}$ Esta cuestión ha sido desarrollada de manera más amplia por Martínez Nicolás (2009).
} 


\section{Las etapas de las ciencias de la comunicación en España: antecedentes (1965-1980), desarrollo (1980-1995) y estandarización (1996-tiempo presente)}

En general, las etapas de la investigación en mi país han sido abordadas por varios autores (Jones, 1999; Rodrigo Alsina, 2001; Martínez Nicolás, 2009; García Jiménez, 2007). Podríamos resumir todas estas divisiones de la siguiente manera:

1. Etapa I. 1970-1980. Antecedentes y fundación de las ciencias de la comunicación en España.

2. Etapa II. 1980-1995. Desarrollo de las ciencias de la comunicación españolas, lo que Martínez Nicolás (2009, p. 5) ha definido como la "agitación en el campo".

3. Etapa III. 1996-2013. Consolidación y estandarización.

El contexto histórico en el que se desarrolla la investigación es de suma importancia (Martínez Nicolás, 2009, p.5), debido a que responde a la pregunta fundacional en la elaboración de la historia intelectual de un campo de estudio. Esta pregunta fundacional es: ¿por qué hicimos lo que hicimos? Es muy probable que en la reconstrucción de nuestro propio pasado investigador, con frecuencia hayamos olvidado conectar las líneas de investigación impulsadas con las coordenadas sociales y mediáticas (momento histórico y estado de la industrias de los medios), académicas (estudios universitarios, revistas, asociaciones, etc.) y epistemológicas (paradigmas de investigación dominantes). Es decir, la revisión de nuestro propio pasado investigador ha mostrado una débil presencia de la labor de interpretación histórica. Pero este aspecto es fundamental si queremos responder al porqué, a la razón de ser, al sentido de nuestra investigación.

El que la investigación comenzara a desarrollarse en España, principalmente, a partir de la década de los 80 , responde al proceso de consolidación de la identidad de la comunicación como disciplina científica a nivel internacional. Recordemos el mítico volumen de Journal of 
Communication publicado en 1983, "the ferment in the field", en el que algunas de las principales figuras del campo reflexionaban sobre la identidad científica de una comunidad epistémica que mostraba ya claros rasgos de madurez. O la publicación, en 1987, del primer Handbook of Communication Science editado por Berger y Chaffee. Desde el punto de vista de la historia intelectual comunicológica, los 80 recogieron los frutos de una investigación que a mediados del siglo XX mostró ya un cierto grado de institucionalización, con la fundación de la International Communicacion Association (ICA); la International Association of Mass Communication Research (IAMCR) auspiciada por la UNESCO; los míticos debates Schramm/ Berelson (1959); o las disputas paradigmáticas y metodológicas entre la Escuela de Frankfurt y la Mass Communication Research (Lazarsfeld, 1941), dos perspectivas que dominaron la investigación hasta la década de los 60 del pasado siglo.

¿Cuál es la conexión de la investigación española con el contexto internacional? Desde este punto de vista local-global, el ejemplo español es un caso paradigmático de aquellas comunidades epistémicas que vieron llegar sus estudios a la universidad en la década de los 70, al igual que sucedió en España (Rodrigo Alsina y García Jiménez, 2010).

En general, antes de la década de los 80 , debido al débil tejido institucional e investigador, no puede hablarse de la existencia de una disciplina comunicativa en sensu stricto. La primera etapa previa a los 80 es el momento en el que se fragua y comienza a emerger el campo español. Pero no será hasta los 80 cuando podamos hablar de un campo de investigación en un sentido más amplio. Veamos algunas de las coordenadas históricas, académicas y epistemológicas de las etapas de investigación en el país ibérico.

\section{Etapa I. Antecedentes y emergencia. 1965-1980}

En lo referente al contexto social e histórico, es en el curso 70-71 el momento en el que las antiguas escuelas de periodismo dieron al fin el salto al ámbito universitario. Las facultades 
de comunicación en Navarra (Universidad de Navarra), Barcelona (Universidad Autónoma) y Madrid (Universidad Complutense) echaron a andar en pleno tardofranquismo, con una dictadura agonizante (Franco muere en 1975) y con una ley de prensa (la conocida como "Ley Fraga" desde 1966 y vigente hasta 1977) que había dado un cierto margen de maniobra a la profesión periodística. Fue un tiempo en el que hubo ciertos acercamientos de las democracias occidentales hacia el régimen (es el caso de las visitas de Kissinger y Nixon). A la vez, la dictadura era criticada por el movimiento obrero, estudiantil y la democracia cristiana, con algunas figuras emblemáticas como la del cardenal Vicente Enrique y Tarancón. El que los estudios en España emergieran en ese contexto de decadencia del antiguo régimen y muy cercano a la transición hacia la democracia, es algo que hemos pensado poco, pero que resulta fundamental de cara a entender los orígenes de nuestra disciplina.

En el panorama de los medios, podría decirse que la paleotelevisión era representativa de un sistema mediático de carácter generalista, con una audiencia heterogénea y caracterizado por las referencias al mundo externo (Eco, 1986). Los medos públicos dominaban el panorama frente a unos medios privados que todavía no habían entrado en procesos ni de globalización ni de transnacionalización.

Con respecto a la academia, hasta el comienzo de lo estudios de comunicación en la universidad, tuvimos poca proliferación científica, ideologizada y homogeneizada por la censura y el control del estado. Es decir, que no es hasta la década de los 70 cuando puede realmente hablarse del nacimiento de las ciencias de la comunicación españolas (García Jiménez, 2007, p. 155).

Las antiguas escuelas de periodismo fueron sustituidas en el curso 1970-1971 por la entrada de los estudios de comunicación en la Universidad. Esto fue clave para impulsar las ciencias de la comunicación en España, dado que las facultades presentaban una mayor autonomía organizativa e intelectual que las antiguas escuelas oficiales (Martínez Nicolás, 2009, p. 3). 
Aquella comunidad científica originaria, estuvo integrada por docentes de escuelas oficiales, licenciados/doctores en humanidades y profesionales de la comunicación, con una marcada escasez de investigadores procedentes de las ciencias sociales clásicas y, por supuesto, investigadores formados desde un estatuto comunicativo propio (Martínez Nicolás, 2009, p. $4)$.

Fue en esta primera etapa el momento en el que se configuraron nuestros estudios en función del periodismo, la publicidad y lo audiovisual, división que arrastra desde sus orígenes no pocas polémicas. A día de hoy, no termina de estar clara la delimitación entre periodismo y comunicación audiovisual y es muy probable que esta última haya sufrido, incluso en mayor medida que el resto de grados, un giro hacia la formación técnica que la hace, en ocasiones, homologable a una formación profesional superior, más que a una formación científica universitaria. Martínez Nicolás (2009, p. 11) señala cómo en la división de las carreras de comunicación en periodismo, audiovisual y publicidad, división exclusivamente española, probablemente "se trató de otorgar a unos y a otros áreas particulares de poder académico".

Por tanto, unos comienzos institucionalmente débiles debido a la escasez de facultades, revistas (principalmente Mensaje y Medios del Instituto Oficial de Radio y Televisión, IORTV), congresos y grupos de investigación en comunicación.

Epistemológicamente, las influencias pioneras procedieron de la publicística alemana, la Escuela de Frankfurt, la Mass Communication Research y la Semiótica (Martínez Nicolás, 2009, p.4). El proceso comunicativo fue concebido de manera estática, el objeto precedió al proceso; frente al dominio en el proceso comunicativo del emisor y la consiguiente desaparición de la figura de la audiencia, se terminó priorizando el análisis de los textos, en una etapa investigadora de "pre-mediaciones".

\section{Etapa II. Desarrollo 1980-1995}


Esta etapa coincide con el periodo democrático. El gobierno recayó en el Partido Socialista Obrero Español (PSOE) desde 1982 hasta 1996. Además, España entró en el mercado común (hoy Unión Europea) en 1986. En la década de los 90 se produjo la desregulación de la televisión, con la emergencia de cadenas privadas y los primeros grupos de comunicación transnacionales (Grupo Prisa, el actual grupo Unedisa, antiguo grupo recoletos, Grupo ZETa etc.) (Martínez Nicolás, 2009, p.5). Aparecieron algunas innovaciones tecnológicas -como el servicio de información del teletexto accesible mediante la pantalla del televisor-, que, aunque no cumplieron las expectativas creadas, sirvieron para comenzar a hablar de la televisión interactiva y de una audiencia que parecía comenzaba a ser participativa. Asimismo, España asistió al uso generalizado de las computadoras e Internet comenzó a

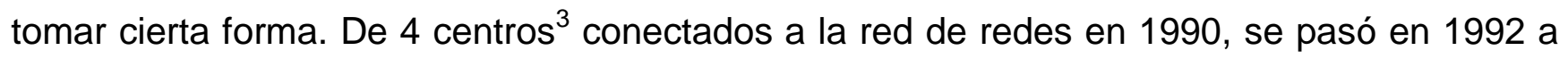
la conexión a Internet por parte de las universidades españolas a través de REDIRIS. En 1993 había en España más de 10.000 ordenadores conectados y ya en el 94, cuando Internet pasó de ser gestionado por REDIRIS a ser gestionado por el CSIC (Rivero, 2001), fueron 20.000 los ordenadores conectados y 100 las organizaciones con plena conectividad.

En lo referente al panorama mediático, la desregulación del mercado televisivo y la consiguiente proliferación de cadenas privadas, desencadenaron la batalla por las audiencias y el paso definitivo a la neotelevisión. Televisión autorreferencial y espectacular (Eco, 1986) que engendraría el periodo de la posmodernidad mediática que vivimos en la contemporaneidad.

Por otra parte, es en esta etapa cuando se produjo la eclosión de las facultades de comunicación en España. De las 3 facultades iniciales (con unos 5000 estudiantes y 500 profesores), se alcanzó en la década de los 90 una veintena de centros universitarios específicos, con 20.000 alumnos matriculados y 2000 profesores de diversas categorías. Un dato representativo de esta eclosión es que desde 1960 a 1979, se defendieron en España

\footnotetext{
de Andalucía y CIEMAT)

Facultad de Ciencias Políticas, Sociales y de la Comunicación Universidad de La Laguna Avenida César Manrique, s/n; Campus de Guajara 38071 La Laguna, Tenerife (Islas Canarias - España)
}

${ }^{3}$ Fundesco, Departamento de Ingeniería Telemática (Universidad Politécnica de Madrid), Centro de Informática Científica 
88 tesis en comunicación. Sin embargo, a partir de 1980 y hasta 1998 fueron 1453 los trabajos doctorales enmarcados en este ámbito de estudio (Martínez Nicolás, 2009, p. 5). Con el aumento de doctores formados específicamente en comunicación, se complejizó el tejido de la comunidad científica. Los docentes con formación en ciencias sociales comenzaron a llegar a las facultades de comunicación y perdieron peso, en la misma medida, el profesorado procedente de las humanidades.

La formación en los grados estuvo orientada a los medios de comunicación, cuestión lógica si tenemos en cuenta cómo las facultades se configuraron desde sus orígenes para responder a la demanda de profesionales de los medios, principalmente. Otros aspectos del mundo profesional de la comunicación (como la organización, lo interpersonal, lo grupal, etc.) quedaron eclipsados frente a los profesionales del mundo del periodismo, la publicidad y el cine y la televisión. En lo referente a la formación doctoral, de la misma manera, la impronta mediática unida a una débil formación metodológica fueron algunas de sus señas de identidad.

El tejido institucional se hizo más denso, con 6 instituciones (asociaciones o grupos de investigación) ${ }^{4}$ y varias revistas de referencia (Analisi, Telos, Comunicación y Sociedad y Comunicar) relacionadas con el estudio de la comunicación.

Con respecto a los paradigmas epistemológicos, continuó la pujanza de la semiótica y llegaron las líneas renovadoras iniciadas en los 70 por las ciencias sociales empíricas, los estudios culturales y la economía política (Martínez Nicolás, 2009, p. 5). Fue el tiempo de las mediaciones (Martín Barbero, 1987), el análisis de los procesos comenzó a desplazar a los objetos. La entrada de los estudios culturales abrió el proceso comunicativo a las audiencias, con lo que se superó en parte la textualidad caracterizadora del estudio de la comunicación más propia de la primera etapa. La comprensión del proceso comunicativo fue, por tanto,

\footnotetext{
${ }^{4}$ Fundesco, la Asociación Hispanoamericana de Centros de Investigación y Empresas de Telecomunicaciones, el CEDIC, el Instituto de Estudios Catalanes, el grupo Comunicar y la Sociedad Española de Periodística.
} 
más compleja que el mero estudio semiótico del texto estático. El modelo sociosemiótico de Rodrigo Alsina (1989) puso el acento en el contexto, en los procesos de producción, en el consumo, en la circulación. Además, los emisores todopoderosos de la Mass Communication Research o la Escuela de Frankfurt comenzaron a tener nombres y apellidos, conexiones con el mundo de la economía y la política e inversores extranjeros. El estudio de los grupos de comunicación desde la Economía Política ahondó en el conocimiento de una figura del proceso comunicativo, los emisores, hasta ese momento prácticamente desconocidos.

\section{Etapa III. Estandarización. 1996-2013}

Es la etapa que estamos viviendo en estos momentos. Ha llegado a la democracia española la alternancia en el poder entre conservadores (Partido Popular, 1996-2004; 2011-presente) y socialistas (PSOE, 2004-2011). Una nueva clase ociosa asiste como usuaria a la revolución de las compañías aéreas de bajo coste, que convierten al turismo en una cuestión de masas, con la consiguiente "museización" de los pueblos subdesarrollados (MacCannell, 2003). Los "no lugares", los lugares de paso, fulgurantes (Augé, 2008), pasan a ser espacios habitables por la gran mayoría y se convierten en la metáfora representativa de las experiencias rápidas, transitorias, que se desvanecen a golpe de nuevas experiencias y relaciones (Bauman, 2003).

En lo concerniente al mundo mediático, los grupos de comunicación transnacionales de la pasada etapa se consolidan y presentan un mayor carácter multimedia. Internet se presenta ya como el paradigma de la sociedad de la información española, con unos índices de conexión que llegan al 50\% de la población, si bien la inserción de la telefonía móvil alcanza a la totalidad de la población del país, siendo su tasa de penetración de 111,5 líneas por cada 100 habitantes $^{5}$. De la neotelevisión espectacular, el digitalismo dará paso a la hipertelevisión (Scolari, 2008, p. 226), un medio que importa/exporta constantemente códigos

\footnotetext{
${ }^{5}$ Fuente: wikipedia. Artículo "Telefonía móvil en España", disponible en http://es.wikipedia.org/wiki/Telefon\%C3\%ADa m\%C3\%B3vil en Espa\%C3\%B1a.
} 
expresivos y visuales de los entornos computacionales y que reenvía a sus usuarios a sus propios contenidos multisoporte. La televisión digital especializa la oferta (con la consiguiente mayor fragmentación de la audiencia que en la era de la neotelevisión). Del modelo broadcasting pasamos definitivamente al modelo basado en el near video on demand, los usuarios pueden recuperar los contenidos e imponer sus propios tiempos de consumo. Se potencia la figura del usuario que consume varios medios a la vez (consumo crossmedia), situación que va a complejizar las mediciones de audiencias y usuarios, con la consiguiente crisis en los modelos de negocio tradicionales mediáticos. Es el tiempo de la cibercultura, esto es, de las nuevas formas de sociabilidad y relación entre individuos, "nuevos sistemas de percepción, acción e interacción social" (Galindo Cáceres, 2006, p.15). Emerge una fuerza de trabajo digital (Scolari, 2008, p. 202) caracterizada por la polivalencia tecnológica, temática y mediática, por la difuminación de los límites profesionales y por la recualificación y flexibilización. Una sociedad red que interconecta, difumina límites, potencia los procesos de horizontalidad y complejiza, con todo ello, los procesos interactivos humanos.

En el mundo académico, de las 20 facultades de la anterior etapa, pasamos a 50 en el año 2013, con un gran aumento de centros de enseñanza privada. Además, el Espacio Europeo de Educación Superior (EEES), integrado por 45 países $^{6}$ del viejo continente, potencia un espacio de educación universitaria homologable y más comprometido con las demandas del mercado, que con las de la sociedad (Lozano y Vicente, 2010, p. 10). La formación tiende a un mayor grado de tecnificación y especialización. Desaparecidos los antiguos estudios de doctorado, se potencia la figura del máster, que, independientemente de su orientación profesional o investigadora, se va a convertir en la puerta que da acceso a la realización de la tesis doctoral. Por otra parte, hay una mayor concienciación de la necesidad de formación metodológica en los estudios de posgrado orientados al mundo de la investigación. Las facultades de comunicación dan un giro de carácter profesionalista que se traduce, en el ámbito de la investigación, en una potenciación de los estudios de carácter empírico de las

${ }^{6}$ Dato actualizado el 23 de septiembre de 2011 en http://www.eees.es/es/eees-paises-participantes.

Facultad de Ciencias Políticas, Sociales y de la Comunicación Universidad de La Laguna 
ciencias sociales, cuantitativos frente a la investigación humanística y formación más teórica de anteriores etapas. El reto es ahora alcanzar un cierto equilibrio entre las demandas del mercado y las de la sociedad y reintroducir en el sistema universitario español objetos de estudio comunicacionales alternativos a la comunicación mediática y digital. Hay una mayoría de profesorado con formación específica en comunicación (Lozano y Vicente, 2010, p. 6), algo que, por otra parte, viene a confirmar las tendencias que en este sentido se producen a nivel internacional (Donsbach, 2006, p.440).

En esta etapa, la red institucional presenta una solidez que no tiene antecedentes en el campo español, con más de 40 publicaciones y grupos de investigación consolidados (García Jiménez, 2007, p. 156-159). La gran mayoría de revistas comienzan a publicarse a finales de los 90; es el caso de Ámbitos (1998), Binaria (2001), Caleidoscopio (2000) o Cuadernos de Información y comunicación (1997). Se trata de proyectos editoriales cuyos autores están adscritos mayoritariamente a universidades -pocos autores no universitarios- y en las que la presencia de América Latina es muy débil (Martínez Nicolás y Saperas, 2009, p. 5). En esta etapa también se produce una mayor proyección internacional de nuestra investigación, proyección al ámbito latinoamericano más que al anglosajón, aunque también se detecta una mayor presencia de investigadores españoles en eventos internacionales (como es el caso de los congresos anuales de la IAMCR o los eventos impulsados desde ECREA).

Otro aspecto clave de consolidación institucional es la inclusión de las ciencias de la comunicación como disciplina dentro del programa nacional de investigación en ciencias sociales a partir de la convocatoria 2004-2007 (García Jiménez, 2007, p. 159).

Además, resultan claves en esta etapa los procesos de estandarización de la investigación y los estudios universitarios. La emergencia de agencias de calidad como la ANECA (Agencia Nacional de Evaluación de la Calidad y Acreditación en España) endurece los requisitos 
institucionales para la promoción profesional en el ámbito académico, que queda sometido a las presiones del "publish or perish", "publica o perece" (Martínez Nicolás, 2009, p. 8).

Apunta Soriano (2008) la aparición del "efecto ANECA", es decir, la valorización de las publicaciones recogidas en los índices de impacto internacionales (como el Social Science Citation Index), con la consiguiente pérdida de valor de la publicación de libros. El "efecto ANECA" supone adoptar para la comunicación, los modelos de evaluación que rigen la investigación en ciencias naturales. Es sobre todo una investigación de corte empirista y sociológico: "El desarrollo de las agencias de evaluación, coincide con el despegue del sector de las revistas académicas, hasta entonces hubo una preponderancia de los libros" (Soriano, 2008, p. 12).

Desde un punto de vista epistemológico, continúa la exploración de los procesos frente a los objetos, procesos complejizados en mayor medida por el paso de las mediaciones (medios tradicionales) a las hipermediaciones (inclusión también de los medios digitales): "Al hablar de hipermediación no nos referimos tanto a un producto o un medio sino a procesos de intercambio, producción y consumo simbólico que se desarrollan en un entorno caracterizado por una gran cantidad de sujetos, medios y lenguajes interconectados tecnológicamente de manera reticular entre sí" (Scolari, 2008, pp. 113 y 114). Las hipermediaciones aumentan la presencia de sujetos y medios y potencian los reenvíos, las contaminaciones, las hibridaciones; en definitiva, nuevas configuraciones que van más allá de los medios tradicionales. Por tanto se trata de un tiempo de mezcla, tiempo de mixturas, en el que "desaparecen los paradigmas férreos, las disciplinas cerradas, las teorías omnicomprensivas, las metodologías excluyentes, las certezas perennes y el conocimiento perdurable" (Martínez Nicolás, 2009, p. 8).

Toma fuerza la idea de la transdisciplinariedad y de integración metodológica y paradigmática. La comunidad científica asiste a la instalación en el imaginario colectivo de las expresiones "multiparadigma", "triangulación metodológica", etc., aunque hay un dominio 
de las metodologías cuantitativas y la investigación empírica de las ciencias sociales. Definitivamente, las perspectivas históricas y humanísticas tienden a desaparecer y aumentan las tecnológicas y científico-sociales (Soriano, 2009).

\section{La producción científica española en función de las etapas}

En general, apunta Castillo (2010, p. 294) que la investigación en España ha pasado de estar dominada por investigadores masculinos, a un cierto equilibrio entre ambos sexos $(53,7 \%$ hombres, frente al 46,3\% mujeres), si bien hay una mayor presencia de investigadores e investigadoras de las universidades con más tradición o que pertenecen a nodos claves del país (Universidad Autónoma de Barcelona, Universidad Complutense, Universidad del País Vasco, Universidad Rey Juan Carlos) (Castillo, 2010, p. 311). Se trata también de una investigación en la que ha aumentado la colaboración entre investigadores pertenecientes a la misma disciplina, aunque sigue siendo escasa la colaboración interdisciplinar; producción científica impulsada desde las universidades, cuando hace una década éstas no alcanzaban ni un $22 \%$ de lo publicado (Castillo, 2010, p. 321).

Partiendo de estas cuestiones, ahora la pregunta a responder es cuáles han sido las principales líneas de investigación que hemos desarrollado en España. Para medir la proliferación científica española en función de las dos principales etapas de nuestra investigación (la del desarrollo y la de la estandarización), he revisado algunas de las revistas $^{7}$ con más trayectoria e índice de impacto ${ }^{8}$ dentro del campo comunicológico y analizado la producción investigadora de libros y capítulos de libro. No he clasificado toda la producción investigadora en general, sino aquella que presentaba un mayor componente

\footnotetext{
${ }^{7}$ Las revistas que han sido revisadas desde su primer número hasta el año 2007 son: Comunicación y Sociedad, Análisi, Cuadernos de información y comunicación, Telos y Zer

8 Índice de impacto según la base de datos INRECS disponible en http://ec3.ugr.es/in-recs/ii/Comunicacionfecha-2009.htm
}

Facultad de Ciencias Políticas, Sociales y de la Comunicación Universidad de La Laguna Avenida César Manrique, s/n; Campus de Guajara 38071 La Laguna, Tenerife (Islas Canarias - España) 
teórico, es decir, aquellos trabajos que no solamente describían o presentaban un estudio de caso sin más, sino que planteaban algún tipo de formulación teórica mediante hipótesis, interpretación de datos, extrapolación de supuestos, utilización de paradigmas y tradiciones, formulación de teorías, etc. Se han explotado estadísticamente un total de 339 referencias (libros, artículos y capítulos de libro) publicadas entre 1980 y 2009.

La clasificación de la muestra se ha llevado a cabo en función de los principales objetos de estudio de la investigación en comunicación, debido a que los niveles de análisis arrojaban poca luz sobre la investigación española (casi la totalidad de la producción se ha desarrollado en un nivel macro-mediático, frente a la escasez de aproximaciones de carácter micro-interpersonal). Los objetos de estudio manejados en la clasificación teórica son los siguientes (García Jiménez, 2007, p. 178 y ss.):

1. Formulación teórica: obras que no incluyen componente empírico.

- Epistemología/Metodología: estudios que abordan supuestos epistemológicos de la investigación en comunicación o cuestiones de carácter metodológico.

- Metateoría: este objeto de estudio hace alusión a los trabajos manualísticos de recopilación de teorías, tradiciones de pensamiento, etc. Es una teoría de teorías, en el sentido planteado por Craig (1999).

2. Teorías sobre el proceso comunicativo: teorías de rango medio (Merton, 1984), es decir, trabajos teóricos que incluyen, en mayor o menor medida, algún tipo de componente empírico. Este segundo gran objeto de estudio queda dividido en los elementos que integran el proceso comunicativo, que son:

- Elementos subjetivos: referido a los actores que intervienen en el proceso (interlocutores, emisores, receptores, audiencias, etc.). 
- Elementos objetivos, objeto de estudio centrado en el análisis de los contenidos, de los mensajes.

- Elementos práxicos, es decir, los efectos generados por los procesos comunicativos (teorías del framing, agenda setting, etc.).

- Aportaciones teóricas generales ( cultura y sociedad): trabajos que no se centran específicamente en un elemento del proceso comunicativo, sino que lo abordan desde una perspectiva más general, haciendo alusión a varios elementos del proceso y llegando hasta los niveles sociales y culturales.

Como toda clasificación, esta división que planteo fuerza la realidad a estudiar, dado que debemos tener en cuenta que la comunicación es una realidad sistémica que une e interrelaciona y que, por lo tanto, no aísla variables o elementos. Teniendo en cuenta este supuesto básico, en la clasificación de la muestra se ha tomado como referencia el principal objeto de estudio, es decir, aquél que articula el trabajo, no contemplándose objetos de estudio secundarios u otras realidades aludidas en la investigación.

En general, tal y como refleja la tabla I -producción científica española (1980-2009)-, la organización de los objetos de estudio de los más a los menos explorados quedaría como sigue: elementos objetivos, metateoría, epistemología/metodología, teorías generales, elementos subjetivos y finalmente los estudios de efectos (elementos práxicos). En general, los análisis sobre los elementos objetivos han "liderado" las ciencias de la comunicación españolas a lo largo de las tres últimas décadas. Además, se ha producido un decrecimiento de la investigación netamente teórica-no empírica (epistemología/metodología y metateoría), en favor de un tipo de investigación centrada en los análisis de campo de los elementos que integran el proceso comunicativo, es decir, aquellos de carácter más empírico y cuya exploración requiere un mayor refinamiento metodológico (los estudios de audiencia y de efectos) o creatividad e imaginación teóricas (aportaciones teóricas generales). Los objetos 
de estudio más explorados decaen y los que históricamente han sido menos explorados tienden a despertar un mayor interés por parte de la comunidad epistémica española, por lo que es previsible una tendencia al equilibrio (también a la heterogeneidad y dispersión) entre las distintas líneas de investigación.

\begin{tabular}{|c|c|c|c|c|}
\hline \multicolumn{5}{|c|}{ Tabla I. PRODUCCIÓN CIENTIFICA ESPAÑOLA (1980-2009) } \\
\hline & & Etapa II & Etapa III & \\
\hline OBJETOS DE ESTUDIO & $\begin{array}{c}1980-2009 \\
(339)\end{array}$ & $\begin{array}{l}1980-1995 \\
(105)\end{array}$ & $\begin{array}{l}1996-2009 \\
(234)\end{array}$ & $\begin{array}{l}\text { Evolución de la } \\
\text { producción (1980- } \\
2009)\end{array}$ \\
\hline Elementos Objetivos & $\begin{array}{l}28,3 \% \\
(96)\end{array}$ & $\begin{array}{l}27.6 \% \\
(29)\end{array}$ & $\begin{array}{l}28,6 \% \\
(67)\end{array}$ & Sube \\
\hline Metateoría & $\begin{array}{l}17,6 \% \\
(60)\end{array}$ & $\begin{array}{l}26.6 \% \\
(28)\end{array}$ & $\begin{array}{l}13,6 \% \\
(32)\end{array}$ & 8 Ваја \\
\hline Epistem/Metodología & $\begin{array}{l}17,4 \% \\
(59)\end{array}$ & $\begin{array}{l}27.6 \% \\
(29)\end{array}$ & $\begin{array}{l}12,8 \% \\
(30)\end{array}$ & $\checkmark$ Ваја \\
\hline Teorías Generales & $\begin{array}{l}16,2 \% \\
(55)\end{array}$ & $\begin{array}{l}10.4 \% \\
(11)\end{array}$ & $\begin{array}{l}18,8 \% \\
(44)\end{array}$ & Sube \\
\hline Elementos Subjetivos & $\begin{array}{l}12,6 \% \\
(43)\end{array}$ & $\begin{array}{l}4.7 \% \\
(5)\end{array}$ & $\begin{array}{l}16,2 \% \\
(38)\end{array}$ & Sube \\
\hline Elementos Práxicos & $\begin{array}{l}7,6 \% \\
(26)\end{array}$ & $\begin{array}{l}2.8 \% \\
(3)\end{array}$ & $\begin{array}{c}9,8 \% \\
(23)\end{array}$ & S Sube \\
\hline Totales & $100 \%$ & $100 \%$ & $100 \%$ & \\
\hline
\end{tabular}

Fuente: elaboración propia

La exploración de los elementos objetivos (tabla II), es decir, el análisis de textos y discursos, es el gran objeto de estudio dentro de la investigación española a lo largo las tres últimas décadas. Los elementos objetivos han despertado el mayor interés en las dos etapas, donde destaca el gran aumento en la publicación de artículos durante los últimos años. Este dato coincide con lo apuntado por Martínez Nicolás y Saperas (2009) acerca del 
dominio de los análisis acerca del periodismo, la información periodística y los contenidos. Habría varias cuestiones que explicarían este interés histórico por los contenidos, concretamente 3.

En primer lugar, recordemos cómo, de los primeros paradigmas de investigación que caracterizan al campo español, dos de ellos, la periodística y la semiótica, están mayormente centrados en el análisis de las estructura narrativas, lingüísticas, discursivas, es decir, en los elemento presentes y latentes de los textos. En segundo lugar, otra cuestión que podría apuntar el interés del contenido frente a otros elementos del proceso comunicativo, es la propia concepción de la comunicación con la que originariamente se fraguó nuestra investigación: la unidireccionalidad y la ausencia de la audiencia como constructora de significados. Si el proceso comunicativo está dominado por un emisor todopoderoso (Escuela de Frankfurt o Mass Communication Research), tiene sentido que el análisis de los mensajes sea suficiente para dar cuenta de lo que acontece en una comunicación más concebida como objeto que como proceso. En tercer y último lugar, metodológicamente, la recuperación y estudio de textos impresos estáticos conlleva una menor complejidad que el análisis de procesos dinámicos y menos apegados a un soporte material (interacciones).

\begin{tabular}{|c|c|c|c|}
\hline \multicolumn{4}{|c|}{ Tabla II. ELEMENTOS OBJETIVOS } \\
\hline & Etapa II & Etapa III & Totales \\
\hline Producto científico & $1980-1995$ & 1996-2009 & $1980-2009$ \\
\hline $\begin{array}{l}\text { Libros y capítulos de } \\
\text { libros }\end{array}$ & $16,6 \%(16)$ & $15,6 \%(15)$ & $32,2 \%(31)$ \\
\hline Artículos & $13,5 \%(13)$ & $54,1 \%(52)$ & $67,7 \%(65)$ \\
\hline TOTALES & $30,2 \%(29)$ & $69,7 \%(67)$ & $100 \%(96)$ \\
\hline
\end{tabular}

Fuente: elaboración propia 
El 17,4\% de lo publicado por los investigadores españoles ha sido recopilación metateórica (tabla III), obras que fueron importantísimas en el inicio de la disciplina. Recordemos la escasa apertura a las corrientes internacionales durante la dictadura franquista, lo que hizo que la metateoría fuera un elemento imprescindible para introducir en España las trayectorias investigadoras comunicativas que hasta los 70 habían seguidos europeos y americanos. El aislamiento durante los orígenes de la disciplina (Etapa I. 1965-1980) provocó que cuando llegaron a España los primeros paradigmas a través de las obras metateóricas, algunos de éstos se encontraban ya en decadencia en campos de estudio más maduros y con más trayectoria que el español. Por ejemplo, la Mass Communication Research perdió vigencia ya en la década de los 60 , por lo que estaba teóricamente muerta cuando entró a España de la mano de Moragas (1979).

Apuntada la importancia de obras como las de Valbuena (1979), Moragas (1979 y 1981) o Benito (1973 y 1975) durante la etapa de desarrollo (1980-1995), como era previsible, la recopilación metateórica desciende 13 puntos en la etapa de estandarización (a partir de 1996). El desarrollo de la metateoría sigue produciéndose más a través de libros (como manuales para nuestros estudios universitarios) que de artículos.

\begin{tabular}{|l|l|l|l|}
\hline \multicolumn{4}{|c|}{ Tabla III. RECOPILACIONES METATEÓRICAS } \\
\hline & Etapa II & Etapa III & Totales \\
\hline Producto científico & $1980-1995$ & $1996-2009$ & $1980-2009$ \\
\hline $\begin{array}{l}\text { Libros y capítulos de } \\
\text { libros }\end{array}$ & $35 \%(21)$ & $30 \%(18)$ & $65 \%(39)$ \\
\hline Artículos & $11,6 \%(7)$ & $23,3 \%(14)$ & $35 \%(21)$ \\
\hline TOTALES & $46,6 \%(28)$ & $53,3 \%(32)$ & $100 \%(60)$ \\
\hline
\end{tabular}

Fuente: elaboración propia 
Sobre la reflexión de carácter más epistémico y/o metodológico, tal y como recoge la tabla IV, se ha producido a lo largo de las dos etapas estudiadas un cambio significativo en la publicación bibliográfica, esto es, de la publicación de un $11,8 \%$ de libros sobre epistemología en la etapa II, se ha pasado a un 33,8\% en la etapa III. Por tanto, la cuestión epistemológica ha ganado peso en la industria editorial pero la ha perdido en la hemerográfica, cuestión que podríamos explicar por el dominio del paradigma postpositivista y la orientación empírica de las revistas de investigación españolas. Tal y como he recogido en el anterior apartado, recordemos cómo a partir de la década de los 90, los procesos de estandarización han impuesto parámetros de medición de calidad extrapolados directamente desde las ciencias naturales (Soriano, 2009). En general, se ha producido un descenso en las reflexiones sobre filosofía de la ciencia y metodología y es previsible que, frente al dominio de lo empírico social, este objeto de estudio continúe en descenso y desarrollándose a través de la industria editorial principalmente.

\begin{tabular}{|l|l|l|l|}
\hline \multicolumn{3}{|c|}{ Tabla IV. EPISTEMOLOGÍA/METODOLOGÍA } \\
\hline & \multicolumn{1}{|c|}{ Etapa II } & Etapa III & Totales \\
\hline Producto científico & $1980-1995$ & $1996-2009$ & $1980-2009$ \\
\hline $\begin{array}{l}\text { Libros y capítulos de } \\
\text { libros }\end{array}$ & $11,8 \%(7)$ & $33,8 \%(20)$ & $45,7 \%(27)$ \\
\hline Artículos & $37,2 \%(22)$ & $16,9 \%(10)$ & $54,2 \%(32)$ \\
\hline TOTALES & $49,1 \%(29)$ & $50,8 \%(30)$ & $100 \%(59)$ \\
\hline
\end{tabular}

Fuente: elaboración propia 
El cuarto objeto de estudio más explorado en el campo español es el de las aproximaciones teóricas generales (ver tabla V), que suponen un $16,2 \%$ de lo producido. Si hasta aquí hemos visto los objetos de estudio más explorados y cuyo volumen de producción científica ha decaído en las últimas décadas (epistemología/metodología y metateoría), tanto las teorías generales como el resto de objetos de estudio contemplados (elementos subjetivos y práxicos) son áreas que presentan un aumento en sus índices de producción a lo largo de las últimas décadas.

Con respecto a las teorías generales (tabla V), como decíamos, este gran y genérico objeto de estudio ha despertado una mayor atención en la segunda etapa y se ha desarrollado mayormente en forma de libros o capítulos de libro (el 65,4\%), lo que vendría a ratificar la tendencia empírica apegada a estudios de caso concretos de la industria hemerográfica española a partir de la etapa de estandarización de control de la calidad científica.

\begin{tabular}{|c|c|c|c|}
\hline \multicolumn{4}{|c|}{ Tabla V. APORTACIONES TEÓRICAS GENERALES } \\
\hline & Etapa II & Etapa III & Totales \\
\hline Producto científico & 1980-1995 & 1996-2009 & $1980-2009$ \\
\hline $\begin{array}{l}\text { Libros y capítulos de } \\
\text { libros }\end{array}$ & $16,3 \%(9)$ & $65,4 \%(36)$ & $81,8 \%(45)$ \\
\hline Artículos & $3,6 \%(2)$ & $14,5 \%(8)$ & $18,1 \%(10)$ \\
\hline TOTALES & $20 \%(11)$ & $80 \%(44)$ & $100 \%(55)$ \\
\hline
\end{tabular}

Fuente: elaboración propia

Por último, los objetos de estudio cuya exploración conlleva una mayor complejidad metodológica como son los elementos subjetivos (emisores y audiencias, tabla VI) y los práxicos (efectos, tabla VII) han sido desarrollados, principalmente, a través de revistas 
científicas y en la etapa III de estandarización. Aunque sus índices de producción son menores (12,6\% elementos subjetivos y $7,6 \%$ elementos práxicos), el creciente interés despertado por estos objetos de estudio en las últimas décadas, viene a ratificar el giro de las ciencias de la comunicación españolas, desde vertientes más históricas y humanísticas en los orígenes de la disciplina, a una mayor impronta de las ciencias sociales empíricas.

Concretamente, como podemos observar en la tabla VI, casi toda la exploración de los elementos subjetivos se ha producido en la III etapa analizada (el $88,3 \%$ ). De entre emisores y audiencias han sido los primeros los que han recibido una mayor atención por parte de la comunidad científica española; las audiencias son un objeto de estudio aún por explorar en España (Grandío, 2007, p. 155). El estudio de ambos aumenta considerablemente en la tercera etapa de la investigación en España, sin duda más empírica que la primera.

Pero, ¿qué explicaría esta desatención de los estudios de audiencia? De los cuatro paradigmas que fundan la investigación española, la Mass Communication Research y la Escuela de Frankfurt directamente no contemplan los procesos de recepción frente al poder que se le atribuye al mensaje y a los emisores. Las otras dos corrientes, periodística y semiótica, están más centradas en los textos que en los actores. Metodológicamente, los análisis de audiencia requieren un mayor refinamiento metodológico (Martínez Nicolás y Saperas, 2009, p. 8) y el uso de técnicas cualitativas, ambas cuestiones (la formación metodológica y el uso de técnicas cualitativas) presentes de manera débil en la comunicología española. Con respecto a la última cuestión apuntada, recordemos que el análisis de las audiencias desde la perspectiva contemporánea de los estudios culturales se centra en "cómo los distintos grupos sociales en función de la edad, el género o la etnia desarrollan distintos sistemas de interpretación" (Rodrigo Alsina, 2001, p 205), de ahí la necesidad de la potenciación cualitativa. En definitiva, ha habido una potenciación de los análisis sobre el emisor, lo que quizá puede ser justificado por el auge de la economía 
política de la comunicación a partir de los 80 justifique esta potenciación en la exploración del emisor a lo largo de las últimas décadas.

\begin{tabular}{l|l|l|l|}
\hline \multicolumn{4}{c}{ Tabla VI. ELEMENTOS SUBJETIVOS } \\
\hline & Etapa II & Etapa III & TOTALES \\
\hline Producto científico & $1980-1995$ & $1996-2009$ & $1980-2009$ \\
\hline $\begin{array}{l}\text { Libros y capítulos de } \\
\text { libros }\end{array}$ & $2,3 \%(1)$ & $13,9 \%(6)$ & $16,2 \%(7)$ \\
\hline Artículos & $9,3 \%(4)$ & $74,4 \%(32)$ & $83,7 \%(36)$ \\
\hline TOTALES & $11,6 \%(5)$ & $88,3 \%(38)$ & $100 \%(43)$ \\
\hline
\end{tabular}

Fuente: elaboración propia

Finalmente, la tabla VII recoge el objeto de estudio menos investigado por la comunidad epistémica española: los efectos. Al igual que en el análisis de los elementos subjetivos, nos encontramos con la casi ausencia durante la II etapa (hasta el $88,4 \%$ se publica en la etapa III) y, en general, con el dominio de los artículos y su consiguiente análisis de carácter empírico.

\begin{tabular}{|c|c|c|c|}
\hline \multicolumn{4}{|c|}{ Tabla VII. ELEMENTOS PRÁXICOS } \\
\hline & Etapa II & Etapa III & Totales \\
\hline Producto científico & 1980-1995 & 1996-2009 & $1980-2009$ \\
\hline $\begin{array}{l}\text { Libros y capítulos de } \\
\text { libros }\end{array}$ & $7,6 \%(2)$ & $11,5 \%(3)$ & $19,2 \%(5)$ \\
\hline Artículos & $3,8 \%(1)$ & $76,9 \%(20)$ & $80,7 \%(21)$ \\
\hline TOTALES & $11,5 \%(3)$ & $88,4 \%(23)$ & $100 \%(26)$ \\
\hline
\end{tabular}

Fuente: elaboración propia

Facultad de Ciencias Políticas, Sociales y de la Comunicación Universidad de La Laguna Avenida César Manrique, s/n; Campus de Guajara 38071 La Laguna, Tenerife (Islas Canarias - España) 


\section{Conclusiones: hacia un espacio común iberoamericano}

A partir de la propia experiencia española, sus contextos epistemológicos e institucionales (apartado II) y sus líneas de investigación (epígrafe III), quisiera cerrar este trabajo con algunas cuestiones que creo claves de cara a potenciar un espacio común iberoamericano que vaya más allá de la organización de puntuales encuentros académicos.

Desde un punto de vista institucional, sería necesario consolidar grupos de investigación que sistematizaran la colaboración España-Portugal-Latinoamérica, mediante el desarrollo de proyectos $y$, en general, programas de investigación que marquen líneas prioritarias de exploración comparativa entre nuestros países. Sin duda, las instituciones y redes ya construidas (como es el caso de la Red Académica Iberoamericana de la Comunicación RAIC- o de la Federación Latinoamericana de Facultades de Comunicación Social FELAFACS-), pueden ser magníficos puntos de partida para ello.

Otro reto en este sentido es conseguir financiación y la implicación de las grandes empresas de comunicación, así como contar con una mayor colaboración de profesionales del mundo de la comunicación en nuestros proyectos. En este punto, el campo de la salud y la comunicación puede ser un buen ejemplo, dado que los grupos de especialización enmarcados en esta área de interés suelen contar con profesionales del mundo de la medicina.

Socialmente, es necesario que no desatendamos las claves éticas que deben inspirar y guiar nuestra investigación para construir un mundo mejor desde la comunicación. En este sentido, el especial de Communication Monographs titulado "¿Ha marcado la diferencia la investigación en comunicación?" (volumen 77, número 4, año 2010) sobre los resultados que ha conseguido la investigación de nuestro campo es toda una declaración de intenciones. ¿Hemos ayudado a construir un mundo mejor? ¿Debe tener el investigador en comunicación un mayor compromiso ético con el mundo en el que opera? 
Algunas de las claves epistemológicas y teóricas que planteo son la necesidad de teorizar el paso contemporáneo de las mediaciones a las hipermediaciones planteadas por Scolari (2008). En este sentido, para hacernos eco de la complejidad de las interacciones generadas en torno y a partir de los medios tradicionales y digitales hará falta potenciar metodologías de carácter cualitativo y estudios comparativos. Así, el reto está ahora en analizar las interacciones comunicativas entre los elementos del nuevo ecosistema comunicativo, interacciones que van desde la conformación del nuevo espacio público, pasando por la figura convergente del prosumer (productor+consumidor), el nuevo hiperconsumo o consumo multimedia/crossmedia y llegando hasta el contexto cultural digital, es decir, hasta el concepto de cibercultura. Necesitamos mantener una actitud contextualista que evite las reducciones del universalismo, por un lado, y del localismo, por el otro.

Finalmente, para potenciar el espacio común referido en este apartado, veo fundamental realizar un mapa de la investigación iberoamericana, escribir nuestra propia historia intelectual, sus etapas, los contextos académicos, sociales, epistemológicos que nos han caracterizado y rastrear nuestras líneas de investigación comunes. Es decir, qué hemos hecho de manera conjunta, cómo lo hemos hecho, por qué lo hemos hecho.

La pregunta fundacional en este punto es: ¿podemos hablar de un espacio iberoamericano que haya superado la multidisciplinariedad y sea transdisciplinar? ¿Nos encontramos en estos momentos en un paso intermedio de colaboración interdisciplinar entre la mutidisciplina y la transdisciplina? Si queremos construir un espacio común iberoamericano, son necesarios hoy más que nunca trabajos metateóricos que sean el punto de partida para la construcción conjunta. Conocernos mejor para actuar en consecuencia en un mundo global y líquido. 


\section{Bibliografía}

Augé, M. (2008). Los no lugares. Espacios del anonimato. Una antropología de la sobremodernidad. Barcelona: Gedisa.

Bauman, Z. (2003). Vida líquida. Barcelona: Paidós.

Benito, A. (1973). Teoría General de la Información. Madrid: Guadiana de Publicaciones.

Benito, A. (1975). Lecciones de teoría general de la información. La comunicación social. Madrid: Tomo 2. Autor - D.L.

Berelson, B. (1959). "The State of Communication Research", en Public Opinion Quarterly, 23(1), pp. 1 a 6.

Cáceres, M. D. y Caffarel, C. (1992). "La comunicación en España: planteamientos temáticos y metodológicos entre 1987 y 1990", en el III Simposio de la Asociación de Investigadores en comunicación del Estado Español (AICE): La investigación en la comunicación. AICE, Madrid, pp. 22-30.

Castillo. A. y Carretón, M.C. (2010). "Investigación en comunicación. Estudio bibliométrico de las revistas de comunicación en España”, en Comunicación y Sociedad, vol. XXIII, núm. 2, pp. 289-327.

Craig, R. T. (1999). "Communication theory as a field", en Communication Theory, num. 9(2), pp. 119-161.

Eco, U. (1986). La estrategia de la ilusión. Barcelona: Lumen.

Galindo Cáceres, J. (2006). Cibercultura. Un mundo emergente y una nueva mirada. México: Instituto Mexiquense de Cultura.

García Avilés, J.A. y García Jiménez, L. (2009). "La enseñanza de Teorías de la Comunicación en España: análisis y reflexión ante la Convergencia de Bolonia", en revista ZER, num. 27, pp. 271-293.

García Jiménez, L. (2007). Las teorías de la comunicación en España: un mapa sobre el territorio de nuestra investigación (1980-2006). Madrid: Tecnos.

Grandío, M. (2007). "Reflexiones sobre el estudio de las audiencias en España. Carencias y retos de futuro", en revista Sphera Publica, num. Especial, pp. 157-172. 
Jones, D. (1997). "Investigació sobre comunicació social a l’Espanya de les autonomies", en revista Análisi. Quaderns de Comunicació i Cultura, no 21, pp. 101-120.

Jones, D. (1998). "Investigación sobre comunicación en España. Evolución y perspectivas", en ZER, $\mathrm{n}^{\circ}$ 5. Disponible en web http://www.ehu.es/zer/zer5/1jones.html. Fecha de consulta: $2 / 05 / 2006$.

Jones, D. (1999). "Investigaciones en España sobre la comunicación iberoamericana", en revista Comunicación y Sociedad, nº 35, Universidad de Guadalajara, pp. 229-268.

Lazarsfeld, P. (1941). "Administrative and critical research", en Peters, J.D y Simonson, P. Mass communication and American social thought. Key texts 1919-1968. pp. 166-174. Boulder: Rowman\&Littlefield publishers.

Lopez Escobar, E., Llamas, J.P. y McCombs, M. (1996). “Una dimensión social de los efectos de los medios de difusión: agenda-setting y consenso", en Comunicación y Sociedad, vol. IX, nํㅗ 1 y 2, Universidad de Navarra, pp. 91-125.

Lozano Ascencio, C. y Vicente Mariño, M. (2010). "La enseñanza universitaria de las teorías de la comunicación en Europa y América Latina", en Revista Latina de Comunicación Social, num. 65, pp. 255-265. Disponible en http://www.revistalatinacs.org/10/art2/898 URJC/20 Lozano.html. Fecha de consulta: 25/09/2011.

MacCannell, D. (2003). El turista. Una nueva teoría de la clase ociosa. Barcelona: Melusina.

Martín Barbero, J. (1987). De los medios a las mediaciones. México: Gustavo Gili.

Martínez Nicolás, M. (2006). "Masa (en situación) crítica. La investigación sobre periodismo en España: comunidad científica e intereses de conocimiento", en Análisi, num. 33, pp. 135-170.

Martínez Nicolás, M. (2009). "La investigación sobre comunicación en España. Evolución histórica y retos actuales", en Revista Latina de Comunicación Social, num 64, pp. 1-14. Disponible en http://www.ull.es/publicaciones/latina/09/art/01 80001 investigacion/Manuel Martinez N icolas.html. Fecha de consulta: 25/09/2011.

Martínez-Nicolás, M. y Saperas-Lapiedra, E. (2009). "Diez años de investigación sobre comunicación en España (1998-2007). Análisis de los artículos publicados en revistas científicas". VII Bienal Iberoamericana de comunicación. 
Merton, R.K. (1984). Teoría y estructuras sociales. México: Fondo de Cultura Económica.

Moragas, M. d. (1979). Sociología de la comunicación de masas. Barcelona: Gustavo Gili.

Moragas, M. d. (1981). Teorías de la Comunicación. Investigaciones sobre medios en América y Europa. Barcelona: Gustavo Gili.

Rivero, R. (2001). "Evolución de Internet en España". Disponible en http://www.elmundo.es/imasd/docs/cursos/masterperiodismo/2002/rivero-master02espana.html . Fecha de consulta: 26/09/2011.

Rodrigo Alsina, M. (1989). Los modelos de la comunicación. Madrid: Tecnos.

Rodrigo Alsina, M. (2001). Teorías de la comunicación. Ámbitos, métodos y perspectivas. Barcelona: Aldea Global.

Rodrigo Alsina, M. y García Jiménez, L. (2010). "Communication theory and research in Spain: a paradigmatic case of a socio-humanistic discipline", en European Journal of Communication, 25(3), pp. 273-286.

Schramm, W. (1959). "The State of Communication Research: Comment", en Public Opinion Quarterly, 23(1), pp. 6-9.

Scolari, C. (2008). Hipermediaciones. Elementos para una teoría de la comunicación digital interactiva. Barcelona: Gedisa.

Valbuena, F. (1979). La Comunicación y sus clases: aplicaciones a diversos campos de la actividad humana. Zaragoza: Luis Vives.

\section{Forma de citar este artículo en bibliografías}

GARCíA-JIMÉNEZ, L. (2016): "Historia y producción científica de la investigación de la comunicación en España (1980-2009)", en Revista PANGEA № 7, páginas 92 a 120. Tenerife: Red Académica Iberoamericana de Comunicación. Recuperado el _ de de 2 de: http://www.revistapangea.org 